\title{
Use of Blood as a Surrogate Model for the Assessment of Visceral Adipose Tissue Methylation Profiles Associated with the Metabolic Syndrome in Men
}

\author{
Frédéric Guénard ${ }^{1,2}$, Yves Deshaies ${ }^{3,4}$, Frédéric-Simon Hould ${ }^{5}$, Stéfane Lebel ${ }^{5}$, André Tchernof ${ }^{2,4}$, Picard Marceau $^{5}$ and Marie-Claude Vohl ${ }^{1,2^{*}}$ \\ ${ }^{1}$ Institute of Nutrition and Functional Foods (INAF), Canada \\ ${ }^{2}$ School of Nutrition, Laval University, Québec, Canada \\ ${ }^{3}$ Québec Heart and Lung Institute, Québec, Canada \\ ${ }^{4}$ Department of Medicine, Laval University, Québec, Canada \\ ${ }^{5}$ Department of Surgery, Laval University, Québec, Canada
}

"Corresponding author: Marie-Claude Vohl, Institute of Nutrition and Functional Foods (INAF), Université Laval, 2440 Hochelaga Blvd, Québec, Canada, Tel: 41865621314676; Fax: 4186565877; E-mail: marie-claude.vohl@fsaa.ulaval.ca

Received date: November 17, 2015; Accepted date: January 6, 2016; Published date: January 12, 2016

Copyright: ๑ 2016 Guénard F, et al. This is an open-access article distributed under the terms of the Creative Commons Attribution License, which permits unrestricted use, distribution, and reproduction in any medium, provided the original author and source are credited.

\section{Abstract}

Epigenetic mechanisms are known to be involved in tissue-specific differentiation. DNA methylation patterns have been shown to be largely conserved across tissues but with variation for specific genes. However, it is unclear whether the variability observed in the methylation profile of a metabolically active tissue is reflected in other sources such as hematopoietic tissue. This study aimed to test blood genome-wide CpG site methylation levels as a surrogate model for visceral adipose tissue (VAT) methylation and to verify whether it appropriately reflects differences in methylation levels found in VAT between men discordant for the metabolic syndrome (MetS). Tissue specimens (VAT and blood samples) were obtained from 16 severely obese individuals discordant for the MetS. CpG sites methylation levels were measured with the Infinium HumanMethylation450 BeadChip and correlations of methylation levels between VAT and blood were computed. Differences in methylation levels between individuals with and without MetS were tested in both tissues. Pathway analysis was conducted for differentially methylated CpG sites common to both tissues. High cross-tissue correlations were observed for VAT and blood $(0.952 \pm 0.014)$ while some CpG sites had significantly different methylation levels in VAT versus blood. Differential methylation analysis between individuals with and without MetS demonstrated a higher number of differentially methylated CpG sites in VAT than in blood ( 11,778 vs. 881 , respectively) with nearly $4 \%$ of differentially methylated sites found in VAT being also represented in blood. Common differentially methylated sites were involved in inflammatory-, lipid- and diabetes-related pathways. These results suggest that blood methylation levels of specific $\mathrm{CpG}$ sites may adequately reflect VAT methylation levels for some of the MetS-related genes, specifically for inflammatory, lipid and glucose metabolism genes.
\end{abstract}

Keywords: Methylation; Visceral adipose tissue; Blood; Metabolic syndrome; Epigenetics; Obesity; Biomarker

\section{Abbreviations \\ BMI: Body Mass Index; BPD-DS: Biliopancreatic Diversion with Duodenal Switch; CIHR: Canadian Institutes of Health Research; CRP C-Reactive Protein; DBP: Diastolic Blood Pressure; HDL-C: HDL- Cholesterol; IUCPQ: Institut Universitaire de Cardiologie et de Pneumologie de Québec; IPA: Ingenuity Pathway Analysis; LDL-C: LDL-Cholesterol; MetS: Metabolic Syndrome; MetS-: Without the MetS; MetS+: With the MetS; MICB: MHC Class I Polypeptide-related sequence $\mathrm{B} ; \mathrm{N}$ : number; NCEP-ATPIII: National Cholesterol Education Program-Adult Treatment Panel III; PCA: Principal Component Analysis; RPTOR: Regulatory associated Protein of MTOR, complex 1; SBP: Systolic Blood Pressure; total-C: total- Cholesterol; TG: Triglycerides; TSS: Transcription Start Site; UTR: Untranslated Region; VAT: Visceral Adipose Tissue}

\section{Introduction}

The metabolic syndrome (MetS) is defined as the clustering of abdominal obesity, impaired glucose tolerance, dyslipidemia, and hypertension [1] increasing the risk of chronic diseases which represent major causes of death worldwide [2]. MetS appears as a polygenic condition affected by environmental factors [3]. Our group previously demonstrated differences in gene expression in visceral adipose tissue (VAT) of men discordant for MetS at genome-wide levels [4], and revealed the influence of genetic variations on gene expression levels of differentially expressed genes [5,6]. Considering that gene expression is affected by both genetic and environmental factors $[7,8]$, evaluation of these two etiological components is needed to get a better understanding of MetS.

Epigenetics is traditionally referred to as heritable changes in gene expression not due to alteration in DNA sequence [9]. Epigenetic marks are involved in the acquisition and maintenance of organized tissues $[10,11]$. Being altered by both genetic and environmental factors, it represents a potential link through which genetics and environment may cause phenotypic variation [12] and may provide a molecular basis for the theory of developmental origin of health and 
Citation: Guénard F, Deshaies Y, Hould FS, Lebel S, Tchernof A, et al. (2016) Use of Blood as a Surrogate Model for the Assessment of Visceral Adipose Tissue Methylation Profiles Associated with the Metabolic Syndrome in Men. J Mol Genet Med 10: 198. doi: 10.4172/1747-0862.1000198

Page 2 of 8

disease[13]. Accordingly, variations in CpG site methylation levels have been associated with transcriptional regulation, cell differentiation, diseases and cancers [3,10,12]. Based on the evidence pointing toward an accumulation of VAT associated with the development of obesity and obesity-related metabolic complications [14], our group previously established methylation profiles in VAT of men with MetS (MetS+) vs. men without MetS (MetS-) [15]. This allowed the identification of differences in CpG site methylation levels between the two groups and the metabolic pathways potentially involved in the development of MetS [15] including cell cycle regulation, immunity and inflammation. These analyses were conducted directly on a metabolically active tissue obtained during bariatric surgery. However, analyses on such relevant tissues samples are sometimes impossible or extremely difficult to collect. As a result, most genome-wide methylation profiles in humans have been performed on DNA extracted from blood, a readily accessible tissue, and tissue-specific DNA methylation was noted for various genes [16-18]. In addition, the relative accessibility of blood offers the possibility of prospective sampling and makes it attractive for the study of dynamic epigenetic changes [19]. Identification of epigenetic features as disease biomarkers in accessible tissues is more suitable for epidemiological and clinical studies and may potentially lead to effective preventive medicine approaches.

Up to now, it is unclear which epigenetic variations observed in a tissue are representative of another and which variations in DNA methylation are tissue specific, reflecting potential functional differences across tissues. Based on the tissue-specific DNA methylation reported for various genes and the relative conservation of DNA methylation patterns across tissues [20,21], we examined the extent to which genome-wide CpG site methylation levels in blood could be used as a surrogate model for VAT methylation levels. In the second stage, we validated whether blood appropriately reflects VAT differences in methylation levels observed in men discordant for the MetS. Furthermore, gene pathway analyses were conducted to clarify the context in which blood methylation is representative of CpG site methylation levels in VAT.

\section{Materials and Methods}

\section{Subject}

VAT was obtained as previously described [22] in patients undergoing biliopancreatic diversion with duodenal switch (BPD-DS) [23]. Tissue and blood samples were obtained from the Biobank of the Institut universitaire de cardiologie et de pneumologie de Québec (IUCPQ) according to institutionally-approved management modalities. Blood samples were collected preoperatively. Patients were selected based on the presence or the absence of the MetS and diagnosis of MetS was determined using the National Cholesterol Education Program Adult Treatment Panel III (NCEP-ATPIII) guidelines when an individual fulfilled three or more criteria [1]. The study was approved by the Université Laval and IUCPQ ethics committees and was performed in accordance with the principles of the Declaration of Helsinki. All participants provided written, informed consent.

Waist girth, resting blood pressure [systolic (SBP) and diastolic (DBP)], plasma lipids [total-cholesterol (total-C), LDL-cholesterol (LDL-C), HDL-cholesterol (HDL-C) and triglycerides (TG)] and fasting glucose concentrations were measured preoperatively using standardized procedures [24]. Body mass index (BMI) was calculated as weight in kilograms divided by height in meters squared.

\section{Genome-wide methylation analysis}

Genomic DNA was extracted from $200 \mathrm{mg}$ of VAT using the DNeasy Blood \& Tissue kit (QIAGEN, Mississauga, Ontario, Canada) and isolated from the blood buffy coat using the GenElute ${ }^{\text {Tx }}$ Blood Genomic DNA kit (Sigma, St Louis, MO, USA). Following quantification of DNA using both NanoDrop Spectrophotometer (Thermo Scientific, Wilmington, DE, USA) and PicoGreen DNA methods, DNA (1 $\mu \mathrm{g})$ was bisulfite converted and quantitative genome-wide methylation analysis was conducted using the Infinium HumanMethylation450 BeadChip (Illumina, San Diego, CA) interrogating more than 485,000 methylation sites at single-nucleotide resolution. The Infinium HumanMethylation450 BeadChip array was designed for genome-wide methylation analysis with coverage targeted across gene regions, and with sites in the promoter region, 5'UTR, first exon, gene body and 3'UTR. Arrays were processed at the McGill University and Génome Québec Innovation Centre (Montréal, Canada) according to the manufacturer's instructions (Illumina, San Diego, CA).

Methylation data were visualized and analyzed using the GenomeStudio $^{\circ}$ software version 2011.1 (Illumina Inc.) and the Methylation Module. Internal control probe pairs were used for data correction (background subtraction and normalization). Infinium ${ }^{\circ}$ HumanMethylation450 BeadChip using two different probe designs to assess methylation levels (Infinium I and Infinium II assays), assayspecific normalization was conducted using internal control probes with the GenomeStudio Methylation Module normalization algorithm. All CpG sites were detected (detection $\mathrm{P}$ value $\mathrm{P}<0.05$ ) in both tissues and all samples fulfilled quality controls (bisulfite conversion, extension, staining, hybridization, target removal, negative and nonpolymorphic control probes). Methylation levels (beta values; $\beta$ ) were estimated as the ratio of signal intensity of the methylated alleles to the sum of methylated and unmethylated intensity signals of the alleles ( $\beta$ value $=\mathrm{C} /(\mathrm{T}+\mathrm{C})$ ). The $\beta$ values varied from 0 (no methylation) to 1 (100\% methylation). The presence of SNPs in CpG sites surrounding regions potentially affecting methylation levels, and polymorphic $\mathrm{CpG}$ sites according to BeadChip annotation and to Chen et al. [25] (CpG sites with SNP underneath or with SNP at the position where singlebase extension occurs) were systematically excluded, leaving 350,066 $\mathrm{CpG}$ sites for statistical analyses. Differences in methylation levels (mean $\beta$ values) were tested between tissues (VAT vs. blood) as well as between MetS+ and MetS- groups for each tissue using Illumina custom model in GenomeStudio software and False Discovery Rate (FDR) -corrected P values were computed.

\section{Pathways analysis}

Analysis of overrepresented pathways from differentially methylated genes identified in both tissues between MetS+ and MetS- groups was conducted using the knowledge base of the Ingenuity Pathway Analysis (IPA) system ${ }^{\circ}$. The list of genes demonstrating differential methylation in both tissues was classified according to pathways. The likelihood that genes from these lists play a role in a specific pathway was computed and $\mathrm{P}$ values were calculated using a right-tailed Fisher's exact test for each pathway. Overrepresented pathways were then obtained from the list submitted.

\section{Statistical analysis}

Clinical data were expressed as mean \pm SD and according to MetS status. Differences in clinical data between MetS+ and MetS- men were 
Citation: Guénard F, Deshaies Y, Hould FS, Lebel S, Tchernof A, et al. (2016) Use of Blood as a Surrogate Model for the Assessment of Visceral Adipose Tissue Methylation Profiles Associated with the Metabolic Syndrome in Men. J Mol Genet Med 10: 198. doi: 10.4172/1747-0862.1000198

Page 3 of 8

tested using Student's t-test and difference in distribution of CpG sites between tissues was assessed using the Chi-square test. Pearson correlations were first computed for all CpG site methylation levels between MetS+ and MetS- groups. Inter-individual pairwise correlations (Pearson correlations) were also calculated between all individuals for each tissue. Second, CpG sites with extreme high $(>90 \%)$ or low methylation $(<10 \%)$ in all samples assessed were removed and correlations described above were calculated for probes with non-extreme methylation levels. CpG sites with extreme methylation levels were excluded based on the fact that CpG sites either completely methylated or unmethylated across individuals may potentially increase correlation levels artificially and mask the effect of CpG sites with tissue-specific methylation [26]. Unsupervised hierarchical clustering based on Euclidean distance and principal component analysis (PCA) were performed to discriminate biological samples by tissues and MetS status using the Cluster 3.0 software and displayed with TreeView [27]. Statistical significance was defined as $\mathrm{P} \leq 0.05$. Statistical analyses for differences in clinical data between MetS+ and MetS- groups were conducted using the SAS software version 9.3 (SAS Institute Inc). Statistics for genome-wide methylation data were calculated in R version 2.14.1 (R Foundation for Statistical Computing).

\section{Results}

\section{Subjects' description}

Subjects included in this study were middle-aged, severely obese men [mean BMI $54.0 \pm 9.8 \mathrm{~kg} / \mathrm{m}^{2}$ ]. All subjects met the MetS criterion for abdominal obesity defined by the NCEP-ATPIII (Table 1).

\begin{tabular}{|l|l|l|}
\hline Characteristics & MetS- & MetS+ \\
\hline Number of subjects & 8 & 8 \\
\hline Age (years) & $31.1 \pm 10.8$ & $37.2 \pm 9.7$ \\
\hline BMI (kg/m ${ }^{2}$ ) & $57.3 \pm 10.7$ & $50.7 \pm 8.1$ \\
\hline Waist girth (cm) & $156.1 \pm 17.8$ & $152.0 \pm 17.2$ \\
\hline Lipid profile & & \\
\hline Total-C (mmol/l) & $4.13 \pm 0.85$ & $5.35 \pm 1.05^{*}$ \\
\hline LDL-C (mmol/l) ${ }^{\mathrm{b}}$ & $2.40 \pm 0.71$ & $3.21 \pm 0.80^{\dagger}$ \\
\hline HDL-C (mmol/l) & $1.26 \pm 0.22$ & $0.86 \pm 0.13^{* *}$ \\
\hline TG (mmol/l) & $1.06 \pm 0.27$ & $3.00 \pm 1.95^{*}$ \\
\hline Total-C / HDL-C & $3.34 \pm 0.72$ & $6.40 \pm 1.92^{* *}$ \\
\hline Blood pressure (mm Hg) ${ }^{\mathrm{a}}$ & & $10.03 \pm 4.89$ \\
\hline SBP & $136.4 \pm 13.0$ & $156.0 \pm 24.2^{\dagger}$ \\
\hline DBP & $80.3 \pm 5.1$ & $92.0 \pm 18.4$ \\
\hline Fasting glucose (mmol/l) & $5.16 \pm 0.38$ & $0.58^{* *}$ \\
\hline CRP (mg/L) ${ }^{\mathrm{c}}$ & $11.32 \pm 8.34$ & \\
\hline
\end{tabular}

Table 1: Study participants' characteristics.

Referring to Table 1, values presented (means \pm SD) are untransformed and unadjusted values. P values obtained using Student t-test: ${ }^{*} \mathrm{P}<0.05 ;{ }^{* *} \mathrm{P}<0.001 .{ }^{\dagger} \mathrm{P}<0.10$. Due to data unavailability for some participants' characteristics, values presented and statistical results are from 8 MetS- and 8 MetS+ except for: ${ }^{\mathrm{a}} 7 \mathrm{MetS}-$ and $8 \mathrm{MetS}+{ }^{\mathrm{b}} 8 \mathrm{MetS}-$ and $7 \mathrm{MetS}+,{ }^{\mathrm{c}} 6 \mathrm{MetS}-$ and $7 \mathrm{MetS}+$.

Classification of these 16 men according to MetS criteria revealed that both groups showed similar BMI and waist girth while higher total-C and TG levels $(\mathrm{P}<0.05$ for both) were observed in MetS+ individuals. The MetS+ group also had lower HDL-C $(\mathrm{P}=0.0006)$ and higher fasting glucose levels ( $\mathrm{P}=0.0008)$.

\section{Global methylation levels}

Briefly, a mean call rate of $99.97 \%$ was obtained for all 32 samples (16 VAT and 16 blood samples) with a similar mean call rate in both tissues (99.97\% in VAT and $99.98 \%$ in blood).
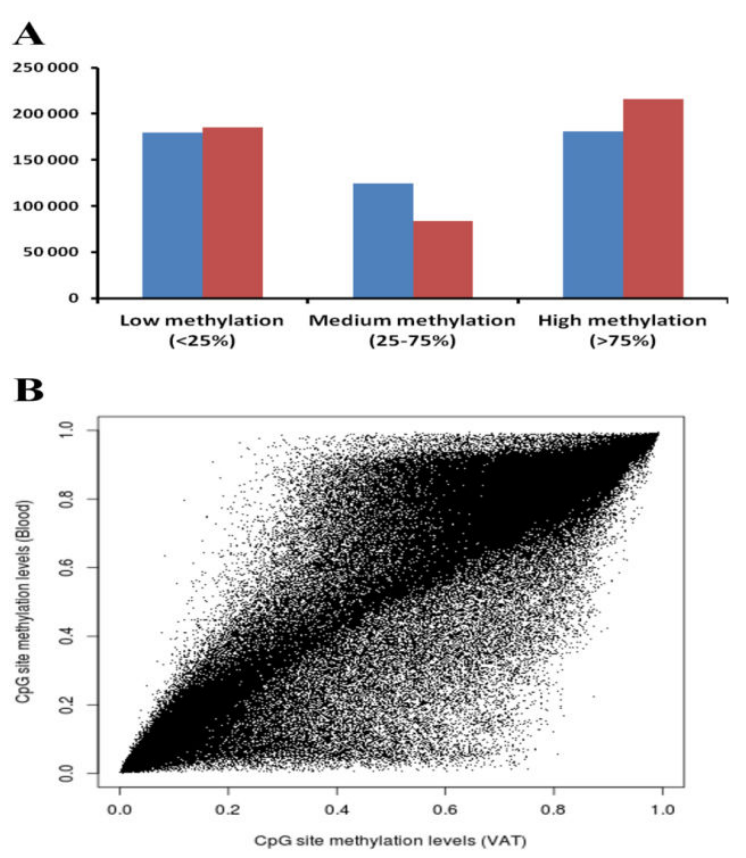

Figure 1: Distribution and correlation of methylation levels. Panel A. Distribution of methylation levels in VAT (blue) and blood (red) for all CpG sites assessed $(\mathrm{N}=485,577)$. Panel B. Correlation of methylation levels between VAT and blood for non-polymorphic CpG sites $(\mathrm{N}=350,066)$. The DNA methylation was measured as $\beta$ value ranging from 0 (unmethylated) to 1 (completely methylated). Abbreviations: N: number; VAT: Visceral Adipose Tissue.

Classification of methylation levels for all sites according to the fraction of probes with low $(<25 \%)$, medium $(\geq 25 \%$ and $\leq 75 \%)$, or high $(>75 \%)$ mean tissue methylation revealed a difference in the distribution of $\mathrm{CpG}$ site methylation levels between tissues (Figure 1, Panel A; $\mathrm{P}<0.0001$ ) with blood demonstrating a lower number of $\mathrm{CpG}$ sites in the medium methylation fraction and higher number of highly (>75\%) methylated sites compared to VAT. Following the exclusion of polymorphic $\mathrm{CpG}$ sites according to BeadChip annotation and to Chen et al. [25], 350,066 CpG sites were used for further analyses. Strong correlations were observed between tissues (Figure 1, Panel B; $r$ $=0.958, \mathrm{P}<2.2 \times 10^{-16}$ ) with the majority of $\mathrm{CpG}$ sites demonstrating similar levels of methylation in both tissues. 
Citation: Guénard F, Deshaies Y, Hould FS, Lebel S, Tchernof A, et al. (2016) Use of Blood as a Surrogate Model for the Assessment of Visceral Adipose Tissue Methylation Profiles Associated with the Metabolic Syndrome in Men. J Mol Genet Med 10: 198. doi: 10.4172/1747-0862.1000198

Page 4 of 8

While high levels of correlation were observed between tissues, 158,226 of the analyzed sites demonstrated statistically significant differences (FDR-corrected $\mathrm{P}$ value $\mathrm{P} \leq 0.05$ ) in methylation levels across tissues. Overall, 65,838 (18.8\%) of the 350,066 CpG sites analyzed showed statistically significant differences in mean methylation levels across tissues greater than 0.1 thus potentially defining tissue-specific methylation patterns. Distribution of these differentially methylated sites demonstrated higher proportions of differentially methylated sites located in gene body and in intergenic regions in comparison to all $\mathrm{CpG}$ sites analyzed (Figure 2).

Inter-tissue correlations were calculated for each individual (16 pairs) and intra-tissue correlations were calculated for all pairs (120 pairs per tissue; each individual against 15 others). Briefly, inter-tissue correlation observed was lower than intra-tissue correlation (Table 2). Taking into account the potential impact of CpG sites with extreme methylation levels on correlation coefficients, a similar analysis was conducted following the exclusion of CpG sites with low $(<10 \%)$ or high $(>90 \%)$ methylation levels. Inter- and intra-tissue correlations between CpG site methylation levels were then calculated for 236,787 sites in both tissues, 219,982 sites in VAT and 216,851 sites in blood. Globally, among non-polymorphic sites, exclusion of sites with extreme methylation levels resulted in lower correlation levels (Table 2) but intra-tissue correlation remained higher than inter-tissue correlation.
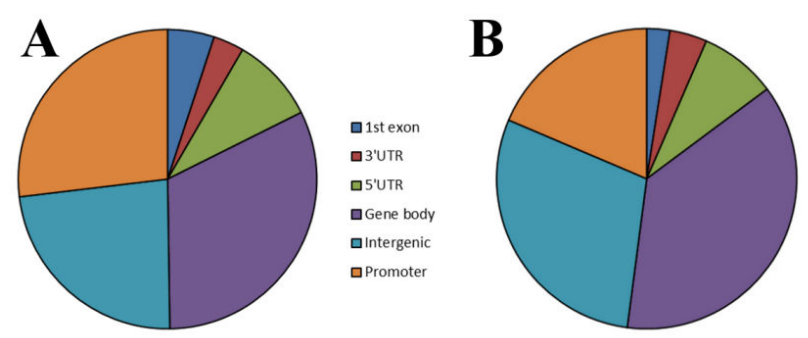

Figure 2: Localization of $\mathrm{CpG}$ sites analyzed. All $\mathrm{CpG}$ sites analyzed (Panel A; $\mathrm{N}=350,066)$ and differentially methylated sites between VAT and blood with difference in methylation levels $>0.10$ (Panel B; $\mathrm{N}=65,838)$ are shown. Abbreviation: $\mathrm{N}$ : number.

\begin{tabular}{|c|c|c|c|c|c|}
\hline \multirow[b]{2}{*}{ Pair } & \multicolumn{2}{|c|}{$\begin{array}{l}\text { All non-polymorphic } \\
\text { sites }(N=350,066)\end{array}$} & \multicolumn{3}{|c|}{$\begin{array}{l}\text { Non-polymorphic sites with } \\
\text { methylation levels between } 0.10 \\
\text { and } 0.90\end{array}$} \\
\hline & $\begin{array}{l}\text { Mean } \\
\text { correlation }\end{array}$ & Range & $\begin{array}{l}\text { Mean } \\
\text { correlati } \\
\text { on }\end{array}$ & Range & $\begin{array}{l}\text { Sites } \\
\text { analyzed }\end{array}$ \\
\hline $\begin{array}{l}\text { VAT vs. } \\
\text { blood }^{a}\end{array}$ & 0.952 & $\begin{array}{l}0.934-0.98 \\
1\end{array}$ & 0.902 & $\begin{array}{l}0.869-0.9 \\
62\end{array}$ & 236,787 \\
\hline Intra-VAT ${ }^{\mathrm{b}}$ & 0.991 & $\begin{array}{l}0.977-0.99 \\
6\end{array}$ & 0.982 & $\begin{array}{l}0.953-0.9 \\
92\end{array}$ & 219,982 \\
\hline Intra-blood ${ }^{b}$ & 0.995 & $\begin{array}{l}0.993-0.99 \\
7\end{array}$ & 0.991 & $\begin{array}{l}0.987-0.9 \\
94\end{array}$ & 216,851 \\
\hline
\end{tabular}

Table 2: Correlation ( $\mathrm{r}$ ) of methylation levels across and intra-tissues. ${ }^{\mathrm{a}} 16$ pairs calculated. ${ }^{\mathrm{b}}$ All pairwise comparison were calculated $(\mathrm{N}=120) .{ }^{c} \mathrm{CpG}$ sites with extreme methylation were excluded based on methylation levels $<10 \%$ or $>90 \%$ in all samples analyzed.
To further confirm higher intra tissue-correlation observed here in comparison to inter-tissue correlation and to visualize structure across and within samples, unsupervised hierarchical clustering and PCA analyses were used. Hierarchical clustering using methylation levels of non-polymorphic CpG sites measured in all samples (350,066 sites) discriminated VAT from blood samples (Figure 3A). Similarly, VAT and blood samples clustered separately in PCA (Figure 3B) thus reinforcing results from correlation analysis showing higher intratissue correlation than inter-tissue correlation. However, none of these analyses allowed discriminating tissue samples according to MetS status.

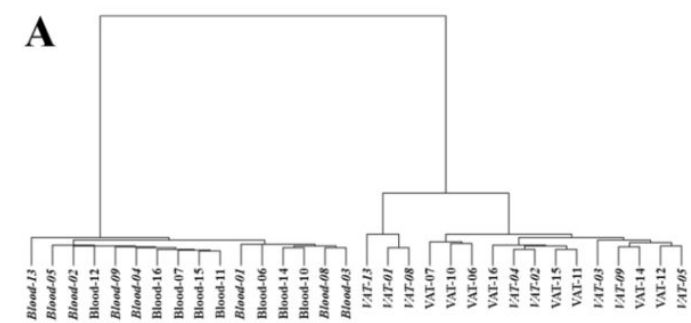

B

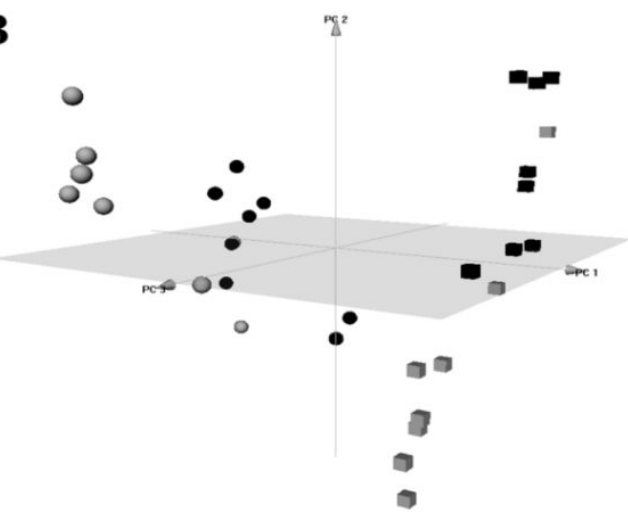

Figure 3: Clustering (Euclidean distance) and Principal component analysis (PCA). Panel A. Samples were clustered according to methylation levels of all sites analyzed $(\mathrm{N}=350,066)$. Tissue samples from MetS+ men are shown in italic. Panel B. Principal component analysis (PCA) on global CpG site methylation levels for nonpolymorphic $\mathrm{CpG}$ sites measured in visceral adipose tissues (VAT) and blood samples $(\mathrm{N}=316,064$ sites $)$ for all 16 individuals. Individuals with (black) and without (grey) the metabolic syndrome are shown. Blood and VAT samples and represented by spheres and cubes respectively. Abbreviations: N: number; MetS+: with the MetS; MetS-: without the MetS.

\section{MetS+ and MetS-group differences}

To assess the use of blood in replacement of metabolically active VAT in studies evaluating methylation levels in relation to cardiometabolic profile, differential methylation analyses between MetS+ and MetS- groups were performed in both VAT and blood for non-polymorphic $\mathrm{CpG}$ sites. A higher number of differentially methylated $\mathrm{CpG}$ sites (FDR-corrected $\mathrm{P}$ value $\mathrm{P} \leq 0.05$ ) were observed in VAT than in blood (Table 3) and represented 4780 and 421 unique genes in VAT and blood, respectively.

Differences in DNA methylation for differentially methylated sites between MetS+ and MetS- groups ranged from -0.26 to 0.39 in VAT 
Citation: Guénard F, Deshaies Y, Hould FS, Lebel S, Tchernof A, et al. (2016) Use of Blood as a Surrogate Model for the Assessment of Visceral Adipose Tissue Methylation Profiles Associated with the Metabolic Syndrome in Men. J Mol Genet Med 10: 198. doi: 10.4172/1747-0862.1000198

Page 5 of 8

and -0.26 to 0.30 in blood. The most differentially methylated sites for both tissues are presented in Table S1. Comparison of the lists of differentially methylated CpG sites (MetS+ vs. MetS-) in both tissues revealed that $437(3.7 \%)$ of the differentially methylated sites in VAT are reflected in blood, while 11,341 differentially methylated CpG sites were VAT-specific and 444 sites were blood-specific.

\begin{tabular}{|c|c|c|}
\hline & VAT & Blood \\
\hline Number of sites analyzed & 350,066 & 350,066 \\
\hline $\begin{array}{l}\text { Differentially methylated CpG sites (FDR- } \\
\text { corrected } \mathrm{P} \text { value } \mathrm{P} \leq 0.05)\end{array}$ & 11,778 & 881 \\
\hline Overmethylated sites in MetS+ & 6075 & 437 \\
\hline Undermethylated sites in MetS+ & 5703 & 444 \\
\hline $\begin{array}{l}\text { Differentially methylated genes (FDR-corrected } \\
P \text { value } P \leq 0.05 \text { ) } C p G \text { site localization* }\end{array}$ & 4780 & 421 \\
\hline Intergenic regions (\%) & $3323(28.1 \%)$ & $329(37.3 \%)$ \\
\hline Gene regions (\%) & $8455(71.8 \%)$ & $552(62.7 \%)$ \\
\hline Gene body & 4685 & 303 \\
\hline 3'UTR & 477 & 32 \\
\hline 1st exon & 265 & 27 \\
\hline 5'UTR & 1109 & 57 \\
\hline Promoter region $^{* *}$ & 1919 & 133 \\
\hline
\end{tabular}

Table 3: Methylation results from the comparison of MetS+ and MetSobese men in VAT and blood.
Referring to Table 3, *Relative to the 1st gene annotated for each CpG site and provided for the Infinium HumanMethylation450 BeadChip. ${ }^{* *}$ Promoter region includes TSS1500 (within 1500-200 bp upstream TSS) and TSS200 (within 200 bp upstream TSS).

Among 437 CpG sites differentially methylated in both tissues, 257 were located in gene regions and represented 203 unique genes including the regulatory associated protein of MTOR, complex 1 (RPTOR) previously associated with obesity [28] and identified among the most differentially methylated genes between MetS+ and MetSgroups in both tissues. Absolute correlation for each of the 437 probes computed from 16 tissue pairs led to a mean correlation of 0.846 across tissues including 434 probes with positive correlations among which 381 showed strong positive ( $r>0.6)$ correlations [29]. Distribution of correlation levels for the 437 probes is shown in Figure S1.

\section{Gene pathway analysis}

The list of differentially methylated sites in MetS+ vs. MetSindividuals common to both VAT and blood was submitted to IPA in order to provide further insights on pathways altered in VAT of MetS+ vs. MetS- also represented in blood. From this list of 203 genes, 15 pathways were significantly overrepresented $(\mathrm{P} \leq 0.05)$ with 19 demonstrating trend for overrepresentation. Among these pathways (Table 4), inflammatory and immune pathways (Antigen presentation, IL-4 signaling) were found with others related to cellular growth and development (BMP signaling, Regulation of Cellular Mechanics by Calpain Protease).

\begin{tabular}{|l|l|l|l|}
\hline IPA canonical pathways & p-value & Diff Meth genes (N) & Total genes in pathway (N) \\
\hline BMP signaling pathway & $3.39 \times 10^{-4}$ & 5 & 77 \\
\hline Antigen Presentation Pathway & $2.95 \times 10^{-3}$ & 3 & 37 \\
\hline Amyloid Processing & $7.76 \times 10^{-3}$ & 3 & 52 \\
\hline Regulation of Cellular Mechanics by Calpain Protease & $1.05 \times 10^{-2}$ & 3 & 58 \\
\hline CDP-diacylglycerol Biosynthesis I & $1.86 \times 10^{-2}$ & 2 & 27 \\
\hline IL-4 Signaling & $2.24 \times 10^{-2}$ & 3 & 77 \\
\hline B Cell Development & $2.69 \times 10^{-2}$ & 2 & 33 \\
\hline Phosphatidylglycerol Biosynthesis II (Non-plastidic) & $2.69 \times 10^{-2}$ & 2 & 33 \\
\hline Altered T Cell and B Cell Signaling in Rheumatoid Arthritis & $3.16 \times 10^{-2}$ & 3 & 88 \\
\hline OX40 Signaling Pathway & $3.24 \times 10^{-2}$ & 3 & 89 \\
\hline Crosstalk between Dendritic Cells and Natural Killer Cells & $3.31 \times 10^{-2}$ & 3 & 90 \\
\hline PAK Signaling & $3.39 \times 10^{-2}$ & 3 & 91 \\
\hline Glutathione-mediated Detoxification & $3.89 \times 10^{-2}$ & 2 & 40 \\
\hline G Beta Gamma Signaling & $4.17 \times 10^{-2}$ & 3 & 98 \\
\hline CDK5 Signaling & $4.68 \times 10^{-2}$ & 3 & 103 \\
\hline Role of NFAT in Regulation of the Immune Response & $5.01 \times 10^{-2}$ & 4 & 177 \\
\hline
\end{tabular}


Citation: Guénard F, Deshaies Y, Hould FS, Lebel S, Tchernof A, et al. (2016) Use of Blood as a Surrogate Model for the Assessment of Visceral Adipose Tissue Methylation Profiles Associated with the Metabolic Syndrome in Men. J Mol Genet Med 10: 198. doi: 10.4172/1747-0862.1000198

Page 6 of 8

\begin{tabular}{|c|c|c|c|}
\hline Neuroprotective Role of THOP1 in Alzheimer's Disease & $5.01 \times 10^{-2}$ & 2 & 46 \\
\hline Triacylglycerol Biosynthesis & $5.13 \times 10^{-2}$ & 2 & 47 \\
\hline Glycerol-3-phosphate Shuttle & $5.37 \times 10^{-2}$ & 1 & 7 \\
\hline Autoimmune Thyroid Disease Signaling & $5.62 \times 10^{-2}$ & 2 & 49 \\
\hline Graft-versus-Host Disease Signaling & $5.62 \times 10^{-2}$ & 2 & 49 \\
\hline Role of NANOG in Mammalian Embryonic Stem Cell Pluripotency & $5.62 \times 10^{-2}$ & 3 & 111 \\
\hline Thioredoxin Pathway & $6.03 \times 10^{-2}$ & 1 & 8 \\
\hline Ga12/13 Signaling & $6.46 \times 10^{-2}$ & 3 & 118 \\
\hline mTOR Signaling & $6.61 \times 10^{-2}$ & 4 & 194 \\
\hline RAR Activation & $6.76 \times 10^{-2}$ & 4 & 197 \\
\hline PKCO, Signaling in T Lymphocytes & $7.08 \times 10^{-2}$ & 3 & 122 \\
\hline Integrin Signaling & $7.24 \times 10^{-2}$ & 4 & 201 \\
\hline Nur77 Signaling in T Lymphocytes & $7.59 \times 10^{-2}$ & 2 & 58 \\
\hline Pyruvate Fermentation to Lactate & $7.59 \times 10^{-2}$ & 1 & 10 \\
\hline S-methyl-5-thio- $\alpha$-D-ribose 1-phosphate Degradation & $8.32 \times 10^{-2}$ & 1 & 11 \\
\hline Insulin Receptor Signaling & $9.55 \times 10^{-2}$ & 3 & 140 \\
\hline Glycerol Degradation I & $9.77 \times 10^{-2}$ & 1 & 13 \\
\hline Calcium-induced T Lymphocyte Apoptosis & $9.77 \times 10^{-2}$ & 2 & 68 \\
\hline
\end{tabular}

Table 4: Pathway analysis from differentially methylated genes. Pathways with trends and significant overrepresentation from genes differentially methylated between MetS+ and MetS- severely obese men identified in both VAT and blood.

Lipid, carbohydrate and amino acid metabolism-related pathways (CDP-diacylglycerol Biosynthesis I, Phosphatidylglycerol Biosynthesis II, Glutathione-mediated Detoxification) were also overrepresented from differentially methylated genes common to VAT and blood. Triacylglycerol Biosynthesis and Insulin Receptor signaling pathways also demonstrated trends toward overrepresentation. Among differentially methylated genes from overrepresented pathway, MHC class I polypeptide-related sequence B (MICB) was previously associated with individual MetS component trait (HDL-C) [30].

\section{Discussion}

Based on the differences in methylation profiles in VAT of men discordant for the MetS previously published by our group [15], the current study assessed global DNA methylation levels in paired tissues from 16 severely obese men discordant for MetS. Using a cohort showing minimal overlap with previously assessed cohort $(\mathrm{N}=2)$ [15], our goal was to investigate whether blood methylation levels may serve as surrogate of VAT methylation levels in the context of an analysis of the determinants of obesity-related metabolic complications. Previous studies assessed methylation levels in paired tissues but few performed inter-tissue analysis in the context of cardiovascular disease risk factors or epigenetic epidemiology $[18,20,21,31,32]$. Testing mean methylation level differences between VAT and blood, the present study reports high correlation levels across tissues. These results are consistent with those previously published in studies evaluating blood and other tissues either focusing on $\mathrm{CpG}$ island or genome-wide methylation levels [20,21,26,33-35], which demonstrated that DNA methylation patterns are globally conserved across tissues. It is important to mention that studies using repetitive elements or focusing on specific CpG sites to assess inter-tissue correlation of genome-wide methylation levels reported lower correlation [36,37], suggesting that assessment method and targets (specific CpG sites across the genome or repetitive elements) impact on the level of correlation observed. Herein, strong correlations were identified in both inter- and intratissue analyses, but lower-magnitude correlations were found in intertissue analyses, consistent with a previous study [20]. Although the current study focused on a cohort of severely obese men discordant for MetS, strong intra-tissue (inter-individual) correlation was found in VAT and blood and compares to intra-tissue correlation reported from other studies $[20,26]$. Despite strong inter-tissue correlation between VAT and blood methylation levels was observed here, differential methylation analysis revealed that $18.8 \%$ of the $\mathrm{CpG}$ sites demonstrated differences in methylation levels greater than 0.1 . These results are reinforced by those obtained from the comparison of the distribution of methylation levels between VAT and blood, showing a higher number of highly methylated CpG sites in blood and with known tissue-specific gene expression. High proportions of differentially methylated sites located in intergenic and gene body regions reported here are consistent with results from mice [38] and humans [31,39] suggesting a role for intragenic and intergenic CpG site methylation in tissue-specific gene expression. Taken as a whole, higher intra- than inter-tissue correlation reported here and in other studies, clustering and PCA analyses, as well as differences in 
Citation: Guénard F, Deshaies Y, Hould FS, Lebel S, Tchernof A, et al. (2016) Use of Blood as a Surrogate Model for the Assessment of Visceral Adipose Tissue Methylation Profiles Associated with the Metabolic Syndrome in Men. J Mol Genet Med 10: 198. doi: 10.4172/1747-0862.1000198

Page 7 of 8

methylation levels between VAT and blood add to the evidence for tissue-specific methylation patterns $[26,31,38,40]$. Differential methylation analysis between MetS+ and MetS- individuals was conducted in both tissues. Higher numbers of differentially methylated CpG sites were observed in VAT than in blood. Differences in cell composition and greater cellular heterogeneity in blood may have contributed to higher variability in sample set and to a lower number of differentially methylated sites. Nonetheless, several of the most differentially methylated sites between MetS+ and MetS- individuals were identified in both tissues. The lists of differentially methylated sites were cross-checked to identify differentially methylated sites in blood reflecting differences observed in VAT. In addition to strong inter-tissue correlation identified for this subset of 437 differentially methylated CpG sites, these results suggest that common differentially methylated sites may have the potential to reveal VAT MetS-associated methylation fingerprint.

Pathway analysis from differentially methylated genes in MetS+ vs. MetS- individuals common to VAT and blood was conducted here to provide insights into VAT functions potentially reflected through blood methylation levels. Alternatively, it may reveal mechanistic alterations common to both tissues in the presence of MetS. Among the most overrepresented pathways according to IPA, lipid and carbohydrate metabolism pathways as well as diabetes pathway were identified in accordance with dyslipidemia and glucose intolerance being components of the MetS [1]. Accordingly, differential expression and methylation of specific diabetes-related genes were reported in VAT $[4,15,41]$ and blood $[42,43]$ of individuals discordant for MetS and specific genes identified here (RPTOR and MICB) were shown to be associated with MetS components [28,30]. Identification of inflammatory genes by differential methylation in both tissues is coherent with the pathogenesis of diabetes as well as with the role of inflammation in the development of MetS $[1,44]$. In addition, these results are in line with systemic inflammation in severely obese individuals with greater deterioration and higher infiltration of immune cells in VAT of MetS+ individuals [44]. Pathways belonging to cellular growth and development categories including BMP signaling, Regulation of cellular mechanics by calpain protease signaling were also overrepresented from the list of differentially methylated genes common to VAT and blood. These genes may potentially reflect cellular mechanisms involved in basal cell function or reveal cellular mechanisms involved in the pathophysiology of MetS. The current study assessed methylation levels in paired tissues from a set of severely obese men with and without MetS. Focusing on a cohort of severely obese men, differentially methylated CpG sites common to VAT and blood identified here may be sex-specific and further analyses in women are thus required. Using a cohort of middle-aged men, the study design limits age-driven changes in methylation reported for specific sites $[45,46]$ and loss of molecular fidelity and ability to maintain methylation patterns across cellular divisions [47]. Exclusion of polymorphic $\mathrm{CpG}$ sites according to BeadChip annotation and to a previous report [25] in the comparison between MetS+ and MetSgroups limits the contribution of the genetic component in the determination of methylation levels. We acknowledge that cellular heterogeneity of tissues assessed here was not taken into account, potentially influencing DNA methylation levels measured. This study focusing on $\mathrm{CpG}$ site methylation levels did not assess gene expression levels. The relationship between gene methylation and expression not being straightforward $[20,21,48]$ and the impact of DNA methylation on gene expression being site- and location-dependent [49] clearly underline the need for further studies on this matter. In addition to establishing the correlation between VAT and blood CpG site methylation levels, the current study identified a set of differentially methylated sites between MetS+ and MetS- severely obese men common to both VAT and blood. Methylation levels of these sites may define a MetS methylation signature in blood and reflect VAT methylation levels for genes in specific pathways involved in the etiology of the MetS. Globally, evidence presented here suggests that for some loci, blood methylation can be used as a surrogate of VAT methylation levels and may potentially reflect VAT dysfunction observed in severely obese men.

\section{Acknowledgment}

We thank all participants in the study for their excellent collaboration, and the members of the department of bariatric surgery for their involvement in clinical care and patient recruitment (Laurent Biertho, Simon Biron, Frédéric-Simon Hould, Stéfane Lebel, Odette Lescelleur, Simon Marceau). We acknowledge the contribution of the biobank staff of the IUCPQ for sample management and of the McGill University and Génome Quebec Innovation Centre for gene methylation array processing. M.C.V. is a Canada Research Chair in Genomics Applied to Nutrition and Health. This study was supported by a grant from the Canadian Institutes of Health Research (CIHR MOP-209380) and by a grant-in-aid from the Heart and Stroke Foundation of Canada (G-14-0005824).

\section{References}

1. Expert Panel on Detection EaToHBCiA (2001) Executive Summary of The Third Report of The National Cholesterol Education Program (NCEP) Expert Panel on Detection, Evaluation, And Treatment of High Blood Cholesterol In Adults (Adult Treatment Panel III). JAMA 285: 2486-2497.

2. Ogden CL, Yanovski SZ, Carroll MD, Flegal KM (2007) The epidemiology of obesity. Gastroenterology 132: 2087-2102.

3. Martinez JA, Cordero P, Campion J, Milagro FI (2012) Interplay of earlylife nutritional programming on obesity, inflammation and epigenetic outcomes. Proc Nutr Soc 71: 276-283.

4. Bouchard L, Tchernof A, Deshaies Y, Marceau S, Lescelleur O, et al. (2007) ZFP36: a promising candidate gene for obesity-related metabolic complications identified by converging genomics. Obes Surg 17: 372-382.

5. Bouchard L, Faucher G, Tchernof A, Deshaies Y, Marceau S, et al. (2009) Association of OSBPL11 gene polymorphisms with cardiovascular disease risk factors in obesity. Obesity.(Silver Spring) 17: 1466-1472.

6. Turcot V, Bouchard L, Faucher G, Garneau V, Tchernof A, et al. (2012) Thymic stromal lymphopoietin: an immune cytokine gene associated with the metabolic syndrome and blood pressure in severe obesity. Clin Sci (Lond) 123: 99-109.

7. Cheung VG, Spielman RS (2009) Genetics of human gene expression: mapping DNA variants that influence gene expression. Nat Rev Genet 10: 595-604.

8. Clement K, Viguerie N, Poitou C, Carette C, Pelloux V, et al. (2004) Weight loss regulates inflammation-related genes in white adipose tissue of obese subjects. FASEB J 18: 1657-1669.

9. Shen L, Waterland RA (2007) Methods of DNA methylation analysis. Curr Opin Clin Nutr Metab Care 10: 576-581.

10. Cantone I, Fisher AG (2013) Epigenetic programming and reprogramming during development. Nat Struct Mol Biol 20: 282-289.

11. Ji H, Ehrlich LI, Seita J, Murakami P, Doi A, et al. (2010) Comprehensive methylome map of lineage commitment from haematopoietic progenitors. Nature 467: 338-342.

12. Feinberg AP (2008) Epigenetics at the epicenter of modern medicine. JAMA 299: 1345-1350. 
Citation: Guénard F, Deshaies Y, Hould FS, Lebel S, Tchernof A, et al. (2016) Use of Blood as a Surrogate Model for the Assessment of Visceral Adipose Tissue Methylation Profiles Associated with the Metabolic Syndrome in Men. J Mol Genet Med 10: 198. doi: 10.4172/1747-0862.1000198

Page 8 of 8

13. Gluckman PD, Hanson MA (2004) Living with the past: evolution, development, and patterns of disease. Science 305: 1733-1736.

14. Tchernof A, Despres JP (2013) Pathophysiology of human visceral obesity: an update. Physiol Rev 93: 359-404.

15. Guenard F, Tchernof A, Deshaies Y, Perusse L, Biron S, et al. (2014) Differential methylation in visceral adipose tissue of obese men discordant for metabolic disturbances. Physiol Genomics 46: 216-222.

16. Barres R, Osler ME, Yan J, Rune A, Fritz T, et al. (2009) Non-CpG methylation of the PGC-1alpha promoter through DNMT3B controls mitochondrial density. Cell Metab 10: 189-198.

17. Ling C, Del GS, Lupi R, Ronn T, Granhall C, et al. (2008) Epigenetic regulation of PPARGC1A in human type 2 diabetic islets and effect on insulin secretion. Diabetologia 51: 615-622.

18. Ribel-Madsen R, Fraga MF, Jacobsen S, Bork-Jensen J, Lara E, et al. (2012) Genome-wide analysis of DNA methylation differences in muscle and fat from monozygotic twins discordant for type 2 diabetes. PLoS One. 7: e51302.

19. Ng JW, Barrett LM, Wong A, Kuh D, Smith GD, et al. (2012) The role of longitudinal cohort studies in epigenetic epidemiology: challenges and opportunities. Genome Biol 13: 246.

20. Byun HM, Siegmund KD, Pan F, Weisenberger DJ, Kanel G, et al. (2009) Epigenetic profiling of somatic tissues from human autopsy specimens identifies tissue- and individual-specific DNA methylation patterns. Hum Mol Genet 18: 4808-4817.

21. Fan S, Zhang X (2009) CpG island methylation pattern in different human tissues and its correlation with gene expression. Biochem Biophys Res Commun 383: 421-425.

22. Vohl MC, Sladek R, Robitaille J, Gurd S, Marceau P, et al. (2004) A survey of genes differentially expressed in subcutaneous and visceral adipose tissue in men. Obes Res 12: 1217-1222.

23. Marceau P, Hould FS, Simard S, Lebel S, Bourque RA, et al. (1998) Biliopancreatic diversion with duodenal switch. World J Surg 22: 947-954.

24. Robitaille J, Despres JP, Perusse L, Vohl MC (2003) The PPAR-gamma P12A polymorphism modulates the relationship between dietary fat intake and components of the metabolic syndrome: results from the Quebec Family Study. Clin Genet 63: 109-116.

25. Chen YA, Lemire M, Choufani S, Butcher DT, Grafodatskaya D, et al. (2013) Discovery of cross-reactive probes and polymorphic CpGs in the Illumina Infinium HumanMethylation450 microarray. Epigenetics 8: 203-209.

26. Ma B, Wilker EH, Willis-Owen SA, Byun HM, Wong KC, et al. (2014) Predicting DNA methylation level across human tissues. Nucleic Acids Res 42: 3515-3528.

27. de Hoon MJ, Imoto S, Nolan J, Miyano S (2004) Open source clustering software. Bioinformatics 20: 1453-1454.

28. Berndt SI, Gustafsson S, Magi R, Ganna A, Wheeler E, et al. (2013) Genome-wide meta-analysis identifies 11 new loci for anthropometric traits and provides insights into genetic architecture. Nat Genet 45: 501-512.

29. Evans JD (1996) Straightforward Statistics for the Behavioral Sciences Brooks/Cole Publishing, US.

30. Kristiansson K, Perola M, Tikkanen E, Kettunen J, Surakka I, et al. (2012) Genome-wide screen for metabolic syndrome susceptibility Loci reveals strong lipid gene contribution but no evidence for common genetic basis for clustering of metabolic syndrome traits. Circ Cardiovasc Genet 5: 242-249.

31. Davies MN, Volta M, Pidsley R, Lunnon K, Dixit A, et al. (2012) Functional annotation of the human brain methylome identifies tissuespecific epigenetic variation across brain and blood. Genome Biol 13: R43.
32. Talens RP, Boomsma DI, Tobi EW, Kremer D, Jukema JW, et al. (2010) Variation, patterns, and temporal stability of DNA methylation: considerations for epigenetic epidemiology. FASEB J 24: 3135-3144.

33. Caliskan M, Cusanovich DA, Ober C, Gilad Y (2011) The effects of EBV transformation on gene expression levels and methylation profiles. Hum Mol Genet 20: 1643-1652.

34. Ursini G, Bollati V, Fazio L, Porcelli A, Iacovelli L, et al. (2011) Stressrelated methylation of the catechol-O-methyltransferase Val 158 allele predicts human prefrontal cognition and activity. J Neurosci 31: 6692-6698.

35. Ronn T, Volkov P, Gillberg L, Kokosar M, Perfilyev A, et al. (2015) Impact of age, BMI and HbA1c levels on the genome-wide DNA methylation and mRNA expression patterns in human adipose tissue and identification of epigenetic biomarkers in blood. Hum Mol Genet 24: 3792-3813.

36. Armstrong DA, Lesseur C, Conradt E, Lester BM, Marsit CJ (2014) Global and gene-specific DNA methylation across multiple tissues in early infancy: implications for children's health research. FASEB J 28: 2088-2097.

37. Wu HC, Wang Q, Chung WK, Andrulis IL, Daly MB, et al. (2014) Correlation of DNA methylation levels in blood and saliva DNA in young girls of the LEGACY Girls study. Epigenetics 9: 929-933.

38. Colaneri A, Wang T, Pagadala V, Kittur J, Staffa NG, Jr, et al. (2013) A minimal set of tissue-specific hypomethylated CpGs constitute epigenetic signatures of developmental programming. PLoS One 8: e72670.

39. Maunakea AK, Nagarajan RP, Bilenky M, Ballinger TJ, D'Souza C, et al. (2010) Conserved role of intragenic DNA methylation in regulating alternative promoters. Nature 466: 253-257.

40. Illingworth R, Kerr A, Desousa D, Jorgensen H, Ellis P, et al. (2008) A novel $\mathrm{CpG}$ island set identifies tissue-specific methylation at developmental gene loci. PLoS Biol 6: e22.

41. Litvinova L, Atochin D, Vasilenko M, Fattakhov N, Zatolokin P, et al. (2014) Role of adiponectin and proinflammatory gene expression in adipose tissue chronic inflammation in women with metabolic syndrome. Diabetol Metab Syndr 6: 137.

42. Ali O, Cerjak D, Kent JW, Jr., James R, Blangero J, et al. (2015) An epigenetic map of age-associated autosomal loci in northern European families at high risk for the metabolic syndrome. Clin Epigenetics 7: 12.

43. Grayson BL, Wang L, Aune TM (2011) Peripheral blood gene expression profiles in metabolic syndrome, coronary artery disease and type 2 diabetes. Genes Immun 12: 341-351.

44. Fuentes E, Fuentes F, Vilahur G, Badimon L, Palomo I (2013) Mechanisms of chronic state of inflammation as mediators that link obese adipose tissue and metabolic syndrome. Mediators Inflamm 2013: 136584.

45. Fraga MF, Agrelo R, Esteller M (2007) Cross-talk between aging and cancer: the epigenetic language. Ann N Y Acad Sci 1100: 60-74.

46. Pirazzini C, Giuliani C, Bacalini MG, Boattini A, Capri M, et al. (2012) Space/population and time/age in DNA methylation variability in humans: a study on IGF2/H19 locus in different Italian populations and in mono- and di-zygotic twins of different age. Aging (Albany NY) 4: 509-520.

47. Goyal R, Reinhardt R, Jeltsch A (2006) Accuracy of DNA methylation pattern preservation by the Dnmt1 methyltransferase. Nucleic Acids Res 34: 1182-1188.

48. Song F, Smith JF, Kimura MT, Morrow AD, Matsuyama T, et al. (2005) Association of tissue-specific differentially methylated regions (TDMs) with differential gene expression. Proc Natl Acad Sci USA 102: 3336-3341.

49. Jones PA (2012) Functions of DNA methylation: islands, start sites, gene bodies and beyond. Nat RevGenet 13: 484-492. 